\title{
Awareness and Attitude among Dental Professional towards CBCT
}

\author{
Keerththana Balabaskaran ${ }^{1}$, Dr.Arathy Srinivasan .L ${ }^{2}$ \\ ${ }^{1}$ (Saveetha Dental college, India) \\ ${ }^{2}$ (Department of oral medicine and radiology, Saveetha Dental College, India)
}

\begin{abstract}
:
Objectives: The present study was carried out to assess the awareness and knowledge of CBCT among practicing general dentists.
\end{abstract}

Materials and methods: A 13 multiple choice questionnaire was given to practicing general dentists to answer. A total of 50 dentists participated in the survey (21 females and 29 males).

Results: Among 50 dentists, about $82 \%(n=41)$ are aware of cone beam computed tomography used for dentomaxillofacial region and $18 \%(n=9)$ are not aware of cone beam computed tomography used for dentomaxillofacial region. out of 41 dentists about 48\% got to know about CBCT through lectures and class, $12 \%$ through internet, 9.7\% through journal, 17\% through seminars, 7.3\% during PG, 2.4\% during trauma case referral and 2.4\% through advertisement. Among 41 dentists about 39\% attended workshops regarding $C B C T$ and $61 \%$ did not attend any workshops. About $80.48 \%$ felt that lower radiation is the most advantage of CBCT over CT. About $87.8 \%$ answered that CBCT offers enhanced diagnosis at lower dose than CT and $7.32 \%$ contradicted this and $4.88 \%$ have no idea. About $14.63 \%$ felt that less radiation is the primary difference between CT and CBCT, 14.63\% felt shape of the beam, 2.44\% cost, and $2.44 \%$ quality and precision and $65.85 \%$ have no idea. About $70.73 \%$ reported that the radiation risk from $C B C T$ is generally higher than conventional CT scans, $9.76 \%$ contradicted this and $19.51 \%$ have no idea. About $68.29 \%$ have referred their patients for CBCT while 31.71\% have not referred. 2.44\% guessed the cost to be less than Rs 500, 26.83\% between Rs $500-1000,17.07 \%$ above Rs. 1000 and 53.66\% having no idea. About 48.78\% reported they would choose CBCT for implant, 35\% for implant and evaluation of cyst and tumors, $19.51 \%$ for all the mentioned cases. About $43.90 \%$ believed that CBCT would be used in the near future in all areas of dentistry, 7.32\% believed that it will not be commonly used in routine practice and $12.2 \%$ have no idea. $39.02 \%$ thought that it is necessary for CBCT to be available at their speciality,56.1\% did not think as necessary and $4.88 \%$ have no idea. Majority of the participants thought that clinical phase should include lectures on CBCT, $14.63 \%$ and $17.07 \%$ on pre clinical and doctoral phase respectively. Majority of the participants about $95.12 \%$ are satisfied with the use of CBCT while $4.88 \%$ are not satisfied.

Conclusion: the present study shows better awareness of CBCT among dental practitioners and this study suggests that more knowledge should be gained on this emerging new technology for better diagnosis and treatment planning

Key words: questionnaire, $C B C T$, radiation

\section{Introduction}

Cone beam computed tomography (CBCT) is an imaging modality that has recently being used useful for dentomaxillofacial imaging. When compared with conventional CT scanners, CBCT unit cost less and require less space, have rapid scan time, reduce the radiation doses ${ }^{1,2,3}$. The drawbacks include beam hardening and scatter from dental materials and poor soft tissue contrast ${ }^{2}$. 3. Common indications for CBCT in dentistry include assessment of the jaws for placement of implants ; examination of teeth and facial structures for orthodontic treatment planning; evaluation of TMJ for osseous degenerative changes, evaluation of mandibular third molar root proximity to mandibular canal prior to extraction; evaluation of teeth and bone for cysts and tumours. ${ }^{4,3}$

\section{Materials And Methods:}

A questionnaire of 13 multiple choice questions were given to general dental practitioners. A total of 50 dentists participated in the study of which $42 \%$ are females and $58 \%$ are males. The questions were about basic difference between $\mathrm{CT}$ and $\mathrm{CBCT}$, radiation, cost and case selection for CBCT.

\section{Questions:}

Are you aware of cone beam computed tomography used for dentomaxillofacial region?

A. Yes

B. No 
If yes proceed to next question.......

1. How did you get to know about CBCT?

2. Do you attend any workshops regarding CBCT?

A. Yes

B. No.

3. Please number the following advantages of CBCT over medical CT from most important (1) to least important(6):

A. Lower radiation dose

B. Shorter scanning time

C. Less expensive

D. Occupies less space

E. Easier to maintain

F. Image processing is easter aue to the limited beam

4. CBCT offers enhanced diagnosis at lower dose than CT?
A. Yes
B. No
C. No idea

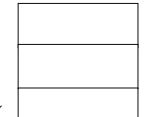

5. what do you think is principal difference between CBCT and CT

6. The radiation dose and risk from CBCT is generally higher than the conventional dental radiography (IOPA, panoramic...)but lower than conventional CT scans
A. Yes
B. No
C. No idea

7. Have you ever referred your patients for CBCT imaging?
A. Yes
B. no

8. What do you think is the cost of CBCT for one image?

9. For what cases would you choose to use CBCT?
A. Implant dentistry
B. Extraction of impacted teeth
C. Evaluation of patients with tumour or cysts
D. Orthodontic assessment
E. All the above
F. Other
G. No need

10. To what extent do you think CBCT will be used in routine dental practice in near future?
A. In all areas of dentistry
B. For selected dental applications $\quad$ which ones?
C. It will not be commonly used in routine practice

D. No idea

11. Do you think it is necessary for a CBCT unit to be available at your speciality?
A. Yes
B. No
C. No idea

12. Which year of dental education should include lectures on CBCT?
A. Preclinical phase
B. Clinical phase
C. Doctoral phase
D. There is no need

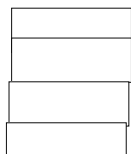

13. Are you satisfied with the use of CBCT?
A. Yes
B. No

III. Results:

The results are summarizing responses to each 13 questions in the survey.

Are you aware of cone beam computed tomography used for dentomaxillofacial region? 
Among 50 dentists, about $82 \%(\mathrm{n}=41)$ are aware of cone beam computed tomography used for dentomaxillofacial region and $18 \%(\mathrm{n}=9)$ are not aware of cone beam computed tomography used for dentomaxillofacial region.

How did you get to know about CBCT?

out of 41 dentists who were aware of CBCT about $48 \%$ got to know about CBCT through lectures and class, $12 \%$ through internet, 9.7\% through journal, $17 \%$ through seminars, $7.3 \%$ during PG, $2.4 \%$ during trauma case referral and $2.4 \%$ through advertisement. $\quad$ ( fig 1 )

Do you attend any workshops regarding CBCT?

Among 41 dentists about 39\% attended workshops regarding CBCT and $61 \%$ did not attend any workshops.( table A)

Please number the following advantages of CBCT over medical CT from most important (1) to least important (6):

About $80.48 \%$ felt that lower radiation is the most advantage of CBCT over CT

(fig 2).

CBCT offers enhanced diagnosis at lower dose than CT?

About $87.8 \%$ answered that CBCT offers enhanced diagnosis at lower dose than $\mathrm{CT}$ and $7.32 \%$ contradicted this and $4.88 \%$ have no idea. ( table A)

what do you think is principal difference between CBCT and CT

About $14.63 \%$ felt that less radiation is the primary difference between $\mathrm{CT}$ and $\mathrm{CBCT}, 14.63 \%$ felt shape of the beam, $2.44 \%$ cost, $2.44 \%$ quality and precision and $65.85 \%$ have no idea. (fig 3 )

The radiation dose and risk from CBCT is generally higher than the conventional dental radiography (IOPA, panaromic...)but lower than conventional CT scans

About $70.73 \%$ reported that the radiation risk from CBCT is generally higher than conventional CT scans, $9.76 \%$ contradicted this and $19.51 \%$ have no idea. ( table A)

Have you ever referred your patients for CBCT imaging?

About $68.29 \%$ have referred their patients for CBCT while $31.71 \%$ have not referred. ( table A)

What do you think is the cost of CBCT for one image?

$2.44 \%$ guessed the cost to be less than Rs $500,26.83 \%$ between Rs $\overline{500-1000,17.07} \%$ above Rs. 1000 and $53.66 \%$ having no idea.

For what cases would you choose to use CBCT ?

About $48.78 \%$ reported they would choose CBCT for implant, 35\% for implant and evaluation of cyst and tumors, $19.51 \%$ for all the mentioned cases.

To what extent do you think CBCT will be used in routine dental practice in near future?

About $43.90 \%$ believed that CBCT would be used in the near future in all areas of dentistry, $7.32 \%$ believed that it will not be commonly used in routine practice and $12.2 \%$ have no idea.

Do you think it is necessary for a CBCT unit to be available at your specialty?

$39.02 \%$ thought that it is necessary for $\mathrm{CBCT}$ to be available at their speciality, $56.1 \%$ did not think as necessary and $4.88 \%$ have no idea. ( table A)

Which year of dental education should include lectures on CBCT?

Majority of the participants thought that clinical phase should include lectures on CBCT , $14.63 \%$ and $17.07 \%$ on pre clinical and doctoral phase respectively.

Are you satisfied with the use of CBCT?

Majority of the participants about $95.12 \%$ are satisfied with the use of CBCT while $4.88 \%$ are not satisfied. (Table A)

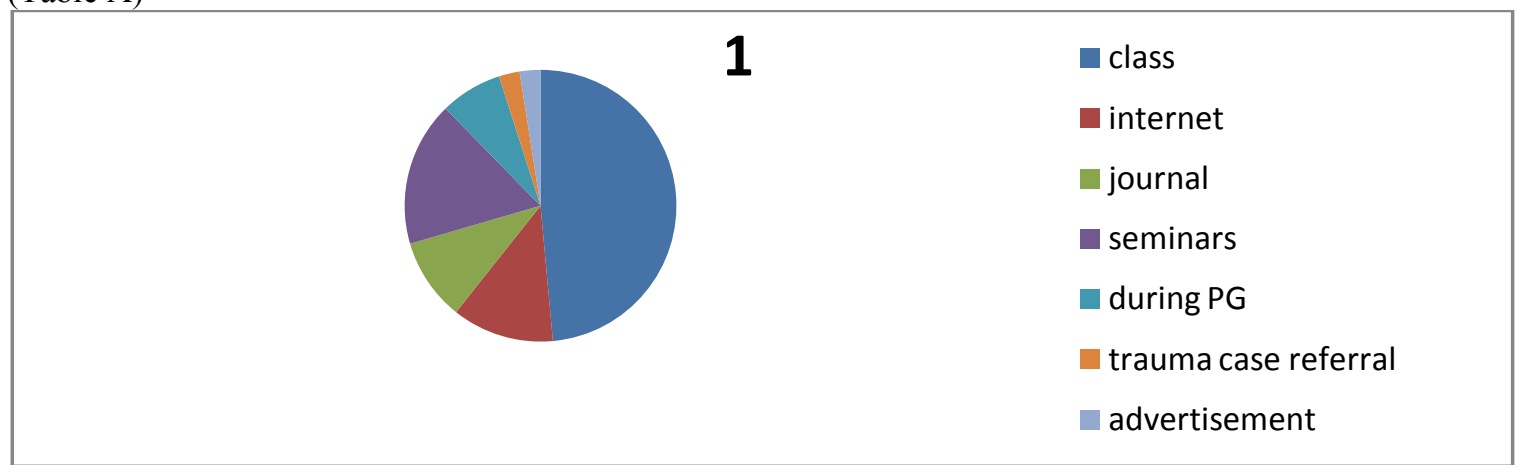




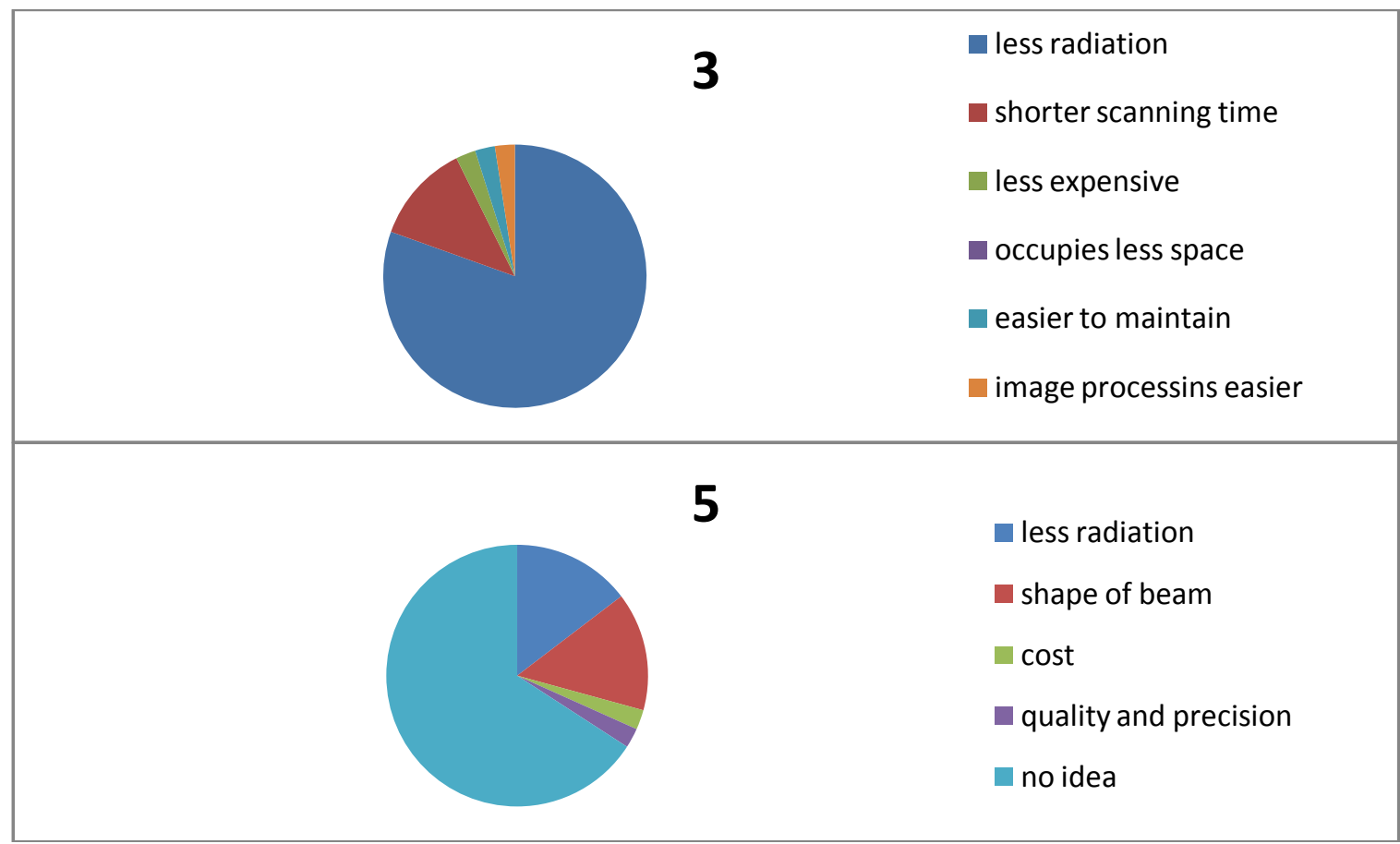

TABLE A:

\begin{tabular}{|l|l|l|l|}
\hline Question & Yes & No & No idea \\
\hline $\begin{array}{l}\text { Do you attend any } \\
\text { workshop regarding } \\
\text { CBCT }\end{array}$ & $39.02 \%$ & $60.98 \%$ & --- \\
\hline $\begin{array}{l}\text { CBCT offers enhanced } \\
\text { diagnosis at lower dose than } \\
\text { CT? }\end{array}$ & $87.80 \%$ & $7.32 \%$ & $4.88 \%$ \\
\hline $\begin{array}{l}\text { 3. Have you ever referred your } \\
\text { patients for CBCT imaging? }\end{array}$ & $68.29 \%$ & $31.71 \%$ & ------ \\
\hline $\begin{array}{l}\text { 4. Do you think it is necessary } \\
\text { for a CBCT unit to be available } \\
\text { at your specialty? }\end{array}$ & $39.02 \%$ & $56.10 \%$ & $4.88 \%$ \\
\hline $\begin{array}{l}\text { 5. Are you satisfied with the use } \\
\text { of CBCT? }\end{array}$ & $95.12 \%$ & $4.88 \%$ & \\
\hline
\end{tabular}

\section{Discussion:}

Studies assessing dental practitioners and students' knowledge about dental radiology have focused mainly on digital systems and radiation protection. While the literature does include one study that evaluates the effectiveness of web-based instruction in the interpretation of anatomy using $\mathrm{CBCT}$ images, no information appears in the literature regarding dental practitioners' knowledge and attitudes about CBCT.

The present study used a questionnaire to gauge the level of knowledge regarding CBCT among dental practitioners.

Among 50 dentists, about $82 \%(\mathrm{n}=41)$ are aware of cone beam computed tomography used for dentomaxillofacial region and $18 \%(\mathrm{n}=9)$ are not aware of cone beam computed tomography used for dentomaxillofacial region. out of 41 dentists about $48 \%$ got to know about CBCT through lectures and class , $12 \%$ through internet, $9.7 \%$ through journal, $17 \%$ through seminars, $7.3 \%$ during PG, $2.4 \%$ during trauma case referral and $2.4 \%$ through advertisement. Among 41 dentists about $39 \%$ attended workshops regarding CBCT and $61 \%$ did not attend any workshops.

About $80.48 \%$ felt that lower radiation is the most advantage of CBCT over CT. About $87.8 \%$ answered that CBCT offers enhanced diagnosis at lower dose than CT and $7.32 \%$ contradicted this and $4.88 \%$ have no idea. By investigating the image quality performance can be quantified. ${ }^{5}$

About $14.63 \%$ felt that less radiation is the primary difference between CT and CBCT, $14.63 \%$ felt shape of the beam, $2.44 \%$ cost, $2.44 \%$ quality and precision and $65.85 \%$ have no idea. About $70.73 \%$ reported that the radiation risk from $\mathrm{CBCT}$ is generally higher than conventional radiographs but less than CT scans, 9.76\% contradicted this and $19.51 \%$ have no idea. Dental practitioners should prescribe CBCT imaging only when they expect that diagnostic yield will benefit patient care, enhance patient safety or improve clinical outcomes significantly ${ }^{6}$. 
About $68.29 \%$ have referred their patients for $\mathrm{CBCT}$ while $31.71 \%$ have not referred. $2.44 \%$ guessed the cost to be less than Rs 500, 26.83\% between Rs 500 - 1000, 17.07\% above Rs. 1000 and 53.66\% having no idea.

About $48.78 \%$ reported they would choose CBCT for implant, 35\% for implant and evaluation of cyst and tumors, $19.51 \%$ for all the mentioned cases. Chau et al compare typical patient radiation doses delivered in implant imaging with spiral computed tomography, conventional spiral tomography and CBCT in their study . they reported that $\mathrm{CBCT}$ delivers lowest radiation dose to organs while spiral multi slice $\mathrm{CT}$ delivers highest dose. $^{7}$

About $43.90 \%$ believed that CBCT would be used in the near future in all areas of dentistry, $7.32 \%$ believed that it will not be commonly used in routine practice and $12.2 \%$ have no idea. $39.02 \%$ thought that it is necessary for $\mathrm{CBCT}$ to be available at their speciality, $56.1 \%$ did not think as necessary and $4.88 \%$ have no idea. Majority of the participants thought that clinical phase should include lectures on CBCT, 14.63\% and 17.07\% on pre clinical and doctoral phase respectively. Majority of the participants about $95.12 \%$ are satisfied with the use of CBCT while $4.88 \%$ are not satisfied.

\section{Conclusion:} tomography.

This study shows the awareness and attitude of dentists towards cone beam computed beam

This high-quality imaging technology should be adopted by dentists, and dental students should be provided with appropriate CBCT education supported by practical experience. More detailed information regarding $\mathrm{CBCT}$ should be included into dental radiology curriculum.

\section{Reference}

[1]. The usage of digital radiography and cone beam computed tomography among Turkish dentists, Dentomaxillofacial Radiology (2011) 40,379-384

[2]. Arai Y, Tammisalo E, Iwai K, Hashimoto K, Shinoda K.Development of a compact computed tomographic apparatus for dental use. Dentomaxillofac Radiol 1999; 28: 245-248.

[3]. Miles DA, Razzano MR. The future of digital imaging in dentistry. Dent Clin North Am 2000; 44: 427-438

[4]. Davies C, Grange S, Trevor MM. Radiation protection practices and related continuing professional education in dental radiography: a survey of practitioners in the North-east of England. Radiography 2005; 11: 255-261.

[5]. R.Pawlels, J. Beinsberger,B. collaert et al; effective dose range for dental scanners; European journal of radiology vol 81, no 2 , pp 267-271, 2012.

[6]. journal of ADA ( Aug 1 2012), 143,899-902

[7]. Chau ACM, Fung K; comparison of radiation dose for implant imaging using conventional spiral tomography, computed tomography, cone beam computed tomography; oral surg oral med oral path oral radiolendo 2009,$107 ; 559-565$

[8]. Versteeg CH, Sanderink GCH, van der Stelt PF. Efficacy of digital intra-oral radiography in clinical dentistry. J Dent 1997; 25: $215-224$.

[9]. Berkhout W, Sanderink G, van der Stelt P. Does digital radiography increase the number of intraoral radiographs? A questionnaire study of Dutch dental practices. Dentomaxillofacial Radiol 2003; 32: 124-127

[10]. Flemish general dental practitioners' knowledge of dental radiology ,Dentomaxillofacial Radiology (2010) 39, 113-118

[11]. Dental students' knowledge and attitudes towards cone beam Computed tomography in Turkey, K Kamburog lu1, S, Kurs, un*,1 and ZZ Akarslan Dentomaxillofacial Radiology (2011) 40, 439-443

[12]. Scarfe WC, Farman AG, Sukovic P. Clinical applications of cone-beam computed tomography in dental practice. J Can Dent Assoc 2006; 72: 75-80.

[13]. Scarfe WC, Farman AG. What is cone-beam CT and how does it work? Dent Clin North Am 2008; 52: 707-730. 